\title{
Fuzzy implications based on semicopulas
}

\author{
Michał Baczyński ${ }^{1}$ Przemysław Grzegorzewski $^{2,3}$ Radko Mesiar $^{4}$ \\ ${ }^{1}$ Institute of Mathematics, University of Silesia, \\ 40-007 Katowice, Bankowa 14, Poland \\ ${ }^{2}$ Systems Research Institute, Polish Academy of Sciences, \\ Newelska 6, 01-447 Warsaw, Poland \\ ${ }^{3}$ Faculty of Mathematics and Computer Science, Warsaw University of Technology, \\ Koszykowa 75, 00-662 Warsaw, Poland \\ ${ }^{4}$ Slovak University of Technology, Radlinskeho 11, \\ 81368 Bratislava, Slovakia
}

\begin{abstract}
Recently, two new families of fuzzy implication functions called probabilistic implications and probabilistic S-implications were introduced by Grzegorzewski $[6,7,9]$. They are based on conditional copulas and make a bridge between probability theory and fuzzy logic. In this paper we generalize these two classes and propose a new kind of construction methods for fuzzy implications which are based on an a priori given fuzzy implication $I$ and a semicopula $B$.
\end{abstract}

Keywords: Copula; Fuzzy implication; Probabilistic implication; Semicopula.

\section{Introduction}

Fuzzy implications play a key role in fuzzy logic and various applications, like approximate reasoning, fuzzy control, fuzzy relational equations, fuzzy mathematical morphology and image processing, etc. In the literature one can find many families of fuzzy implications along with their properties and applications. For the overview of this class of functions see the monograph [3] and the very recent book [1].

Recently, Grzegorzewski [6, 7, 9] introduced two new families of fuzzy implications, abased on copulas - probabilistic implications and probabilistic Simplications. Further investigations on these implications were conducted in [2, 8]. Another family of fuzzy implication operators based on the conditional version of a copula function was proposed by Dolati, Sánchez and Úbeda-Flores [4].

As it is known, copula is a particular case of semicopula, which generalizes also such notions like a quasi-copulas or a t-norm. Therefore, it seems interesting and important to consider also fuzzy implications based on semicopulas.

The paper is organized as follows. In Section 2 we recall basic concepts and definitions used in the paper. In Section 3 we introduce a rather general construction method for implications based on fuzzy implications and semicopulas. Finally, some concluding remarks are added.

\section{Preliminaries}

In this section we recall basic notations and facts used in the sequel.

Definition 2.1 (see [3]). A function $I:[0,1]^{2} \rightarrow$ $[0,1]$ is called a fuzzy implication if it satisfies the following conditions

(I1) $I$ is non-increasing with respect to the first variable,

(I2) $I$ is non-decreasing with respect to the second variable,

(I3) $I(0,0)=I(1,1)=1$ and $I(1,0)=0$.

The family of all fuzzy implications will be denoted by $\mathcal{F} \mathcal{I}$.

We can easily deduce, that each fuzzy implication $I$ is constant for $x=0$ and for $y=1$, i.e., $I$ satisfies the following properties, called left and right boundary conditions, respectively:

$$
\begin{array}{ll}
I(0, y)=1, & y \in[0,1], \\
I(x, 1)=1, & x \in[0,1] .
\end{array}
$$

Therefore, $I$ satisfies also the normality condition $I(0,1)=1$. Consequently, every fuzzy implication restricted to the set $\{0,1\}^{2}$ coincides with the classical implication.

Definition 2.2 (cf. [3]). We say that a fuzzy implication $I$ satisfies the identity principle if

$$
I(x, x)=1, \quad x \in[0,1] .
$$

Definition 2.3 (see [10]). A function $T:[0,1]^{2} \rightarrow$ $[0,1]$ is called a triangular norm (t-norm) if it satisfies the following conditions for all $x, y, z \in$ $[0,1]$

(T1) $T(x, y)=T(y, x)$,

(T2) $T(x, T(y, z))=T(T(x, y), z)$,

(T3) $T(x, y) \leq T(x, z)$ for $y \leq z$, i.e. $T(x, \cdot)$ is non-decreasing,

(T4) $T(x, 1)=x$. 
Definition 2.4 (see [10]). A function $S:[0,1]^{2} \rightarrow$ $[0,1]$ is called a triangular conorm (t-conorm) if it satisfies the following conditions for all $x, y, z \in$ $[0,1]$

(S1) $S(x, y)=S(y, x)$,

(S2) $S(x, S(y, z))=S(S(x, y), z)$,

(S3) $S(x, y) \leq S(x, z)$ for $y \leq z$, i.e. $S(x, \cdot)$ is non-decreasing,

(S4) $S(x, 0)=x$.

Definition 2.5 (cf. [3]). A function $I:[0,1]^{2} \rightarrow$ $[0,1]$ is called an $\mathbf{R}$-implication, if there exists a t-norm $T$ such that

$$
I(x, y)=\sup \{t \in[0,1] \mid T(x, t) \leq y\},
$$

for all $x, y \in[0,1]$. If an R-implication is generated from a t-norm $T$, then we will often denote it by $I_{T}$.

Theorem 2.6 ([11], see also [3, Theorem 2.5.17]). For a function $I:[0,1]^{2} \rightarrow[0,1]$ the following statements are equivalent:

(i) I is an R-implication generated from a leftcontinuous t-norm.

(ii) $I$ is non-decreasing with respect to the second variable, it satisfies the exchange principle, i.e., for all $x, y, z \in[0,1]$

$$
I(x, I(y, z))=I(y, I(x, z)),
$$

it satisfies the ordering property, i.e., for all $x, y \in[0,1]$

$$
x \leq y \Longleftrightarrow I(x, y)=1,
$$

and $I$ is right continuous with respect to the second variable.

Definition 2.7 (see [10]). A non-increasing function $N:[0,1] \rightarrow[0,1]$ is called a fuzzy negation if $N(0)=1, N(1)=0$. Moreover, a fuzzy negation $N$ is called

(i) strict if it is strictly decreasing and continuous;

(ii) strong if it is an involution, i.e. $N(N(x))=x$ for all $x \in[0,1]$.

Definition 2.8 ([12]). A copula (specifically, a 2copula) is a function $C:[0,1]^{2} \rightarrow[0,1]$ which satisfies the following conditions

(C1) $C(x, 0)=C(0, y)=0$, for all $x, y \in[0,1]$,

(C2) $C(x, 1)=x$, for all $x \in[0,1]$,

(C3) $C(1, y)=y$, for all $y \in[0,1]$,

(C4) $C\left(x_{2}, y_{2}\right)-C\left(x_{2}, y_{1}\right)-C\left(x_{1}, y_{2}\right)+C\left(x_{1}, y_{1}\right) \geq$ 0 , for all $x_{1}, x_{2}, y_{1}, y_{2} \in[0,1]$ such that $x_{1} \leq$ $x_{2}, y_{1} \leq y_{2}$.

A family of functions from $[0,1]^{2}$ to $[0,1]$ that generalize copulas are quasi-copulas.
Definition 2.9 ([12]). A function $Q:[0,1]^{2} \rightarrow[0,1]$ which satisfies conditions $(\mathbf{C 1})-(\mathbf{C} 3)$ and

(C4') for all $x_{1}, x_{2}, y_{1}, y_{2} \in[0,1]$ such that $x_{1} \leq x_{2}, y_{1} \leq y_{2}$ it holds

$Q\left(x_{2}, y_{2}\right)-Q\left(x_{2}, y_{1}\right)-Q\left(x_{1}, y_{2}\right)+Q\left(x_{1}, y_{1}\right) \geq 0$,

where at least one of $x_{1}, x_{2}, y_{1}, y_{2} \in\{0,1\}$,

is called a quasi-copula.

It is worth noting that condition $\left(\mathbf{C 4}^{\prime}\right)$ is equivalent to requiring that quasi-copulas are nondecreasing in each variable, i.e. for all $x_{1}, x_{2}, y_{1}, y_{2} \in[0,1]$ such that $x_{1} \leq x_{2}, y_{1} \leq y_{2}$

$$
Q\left(x_{1}, y_{1}\right) \leq Q\left(x_{2}, y_{2}\right),
$$

and satisfy the 1-Lipschitz property, i.e.

$$
\left|Q\left(x_{1}, y_{1}\right)-Q\left(x_{2}, y_{2}\right)\right| \leq\left|x_{1}-x_{2}\right|+\left|y_{1}-y_{2}\right|,
$$

for all $x_{1}, x_{2}, y_{1}, y_{2} \in[0,1]$.

It is clear that every copula is a quasi-copula. Quasi-copulas that are not copulas are called proper quasi-copulas. The family of all quasicopulas will be denoted by $\mathcal{Q C}$.

Another interesting family of functions is given by the semicopulas.

Definition 2.10 ([5]). A function $B:[0,1]^{2} \rightarrow$ $[0,1]$ is called a semicopula if it satisfies conditions (C2)-(C3) and (ND).

The family of all semicopulas will be denoted by $\mathcal{S C}$.

By Definition 2.10 we have

$$
\begin{aligned}
& 0 \leq B(x, 0) \leq B(1,0)=0, \\
& 0 \leq B(0, y) \leq B(0,1)=0,
\end{aligned}
$$

which shows that each semicopula satisfies condition $(\mathbf{C} 1)$.

It is worth noting that the notion of semicopula generalizes some concepts mentioned above. The proof of the following proposition is immediate.

Proposition 2.11 ([5]). (i) A semicopula $C$ which is 2-increasing, i.e., satisfying condition (C4), is a copula.

(ii) A semicopula $Q$ which satisfies the 1-Lipschitz property (Lip) is a quasi-copula.

(iii) A semicopula which is both commutative (T1) and associative (T2) is a t-norm.

It can be shown (see [5, Proposition 2.1]) that each semicopula is bounded, i.e., for any semicopula $B$ and for all $x, y \in[0,1]$ the following inequalities hold

$$
T_{D}(x, y) \leq B(x, y) \leq T_{M}(x, y)
$$

where

$$
T_{D}(x, y)= \begin{cases}0 & \text { if } \quad x, y \in[0,1) \\ \min \{x, y\} & \text { elsewhere }\end{cases}
$$


is the so-called drastic product and

$$
T_{M}(x, y)=\min \{x, y\} .
$$

The notion of conditional copula was applied for defining probabilistic implications and probabilistic S-implications.

Definition $2.12([9])$. Let $C$ be a copula. A function $I_{C}:[0,1]^{2} \rightarrow[0,1]$ given by

$$
I_{C}(x, y)=\left\{\begin{array}{lll}
1 & \text { if } & x=0 \\
\frac{C(x, y)}{x} & \text { if } & x>0
\end{array}\right.
$$

is called a probabilistic implication (based on a copula $C$ ).

Definition $2.13([9])$. Let $C$ be a copula. A function $\tilde{I}_{C}:[0,1]^{2} \rightarrow[0,1]$ given by

$$
\tilde{I}_{C}(x, y)=C(x, y)-x+1,
$$

is called a probabilistic S-implication (based on a copula $C$ ).

Please note that the family $\mathcal{F} \mathcal{I}$ is convex (see $[3$, Theorem 6.2.2]) and the same holds for probabilistic implications and probabilistic S-implications. Indeed, for any $\lambda \in[0,1]$ and for any two probabilistic implications $I_{C_{1}}, I_{C_{2}}$ based on copulas $C_{1}$ and $C_{2}$, a function $K=\lambda I_{C_{1}}+(1-\lambda) I_{C_{2}}$ is given by

$$
\begin{aligned}
K(x, y) & =\left(\lambda I_{C_{1}}+(1-\lambda) I_{C_{2}}\right)(x, y) \\
& =\lambda I_{C_{1}}(x, y)+(1-\lambda) I_{C_{2}}(x, y) \\
& =\left\{\begin{array}{lll}
1 & \text { if } x=0, \\
\frac{\lambda C_{1}(x, y)+(1-\lambda) C_{2}(x, y)}{x} & \text { if } x>0 .
\end{array}\right.
\end{aligned}
$$

Since any convex linear combination of copulas is a copula (see [12]), our function $K$ is a probabilistic implication, which is the desired conclusion. The similar reasoning applies to probabilistic Simplications.

In the next section we will discuss the possibility of utilizing semicopulas for constructing fuzzy implications.

\section{Semicopula based implications}

Assume that $I$ is a fuzzy implication and $B$ is a semicopula. Let us consider a function $J_{I, B}:[0,1]^{2} \rightarrow[0,1]$ based on $I$ and $B$ defined as follows

$$
J_{I, B}(x, y)=I(x, B(x, y)) .
$$

One can easily see that the following proposition holds.

Proposition 3.1. For any fuzzy implication $I$ and any semicopula $B$ a function $J_{I, B}:[0,1]^{2} \rightarrow[0,1]$ defined by (7) satisfies the following conditions:

(i) $J_{I, B}$ is non-decreasing with respect to the second variable, (ii) $J_{I, B}(0, y)=1$,

(iii) $J_{I, B}(1, y)=I(1, y)$,

(iv) $J_{I, B}(x, 0)=I(x, 0)$,

(v) $J_{I, B}(x, 1)=I(x, x)$.

As semicopulas are more general notions than copulas, similarly functions $J_{I, B}$ defined by (7) may be perceived as a generalization of implications based on copulas, i.e., probabilistic implications and probabilistic S-implications. Indeed, the following propositions hold, for which the proofs are straightforward.

Proposition 3.2. Let $I=I_{\mathbf{G G}}$ be the Goguen implication, i.e.,

$$
I_{\mathbf{G G}}(x, y)=\left\{\begin{array}{lll}
1 & \text { if } & x \leq y \\
\frac{y}{x} & \text { if } & x>y
\end{array}\right.
$$

and let $C$ denote a copula. Then $J_{I_{\mathrm{GG}}, C}$ is given by

$$
J_{I_{\mathrm{GG}}, C}(x, y)= \begin{cases}1 & \text { if } x=C(x, y), \\ \frac{C(x, y)}{x} & \text { if } x>C(x, y),\end{cases}
$$

and hence $J_{I_{\mathrm{GG}}, C}$ is a probabilistic implication.

Proposition 3.3. Let $I=I_{\mathbf{L K}}$ be the Eukasiewicz implication, i.e.,

$$
\begin{aligned}
I_{\mathbf{L K}}(x, y) & =\min \{1,1-x+y\} \\
& = \begin{cases}1 & \text { if } x \leq y, \\
1-x+y & \text { if } x>y,\end{cases}
\end{aligned}
$$

and let $C$ denote a copula. Then $J_{I_{\mathbf{L K}}, C}$ is given by

$$
\begin{aligned}
& J_{I_{\mathbf{L K}}, C}(x, y)=\left\{\begin{array}{lll}
1 & \text { if } & x=C(x, y), \\
1-x+C(x, y) & \text { if } & x>C(x, y),
\end{array}\right. \\
& =C(x, y)-x+1
\end{aligned}
$$

and hence $J_{I_{\mathrm{LK}}, C}$ is a probabilistic S-implication.

It is known that a probabilistic implication is not necessarily a fuzzy implication, while each probabilistic S-implication is a fuzzy implication. Therefore, by Propositions 3.2 and 3.3 we conclude that $J_{I, C}$ may be or may not be a fuzzy implication. Looking for the necessary and sufficient criteria required for (7) to obtain a fuzzy implication we get immediately the following theorem.

Theorem 3.4. A function $J_{I, B}:[0,1]^{2} \rightarrow[0,1]$ defined by (7) is a fuzzy implication if and only if it satisfies (I1).

Proof. Let us assume that $J_{I, B}$ given by (7) is a fuzzy implication. In particular it satisfies (I1).

Now let us assume that a function $J_{I, B}$ given by (7) satisfies (I1). From Proposition 3.1 it always satisfies (I2) and

$$
\begin{aligned}
& J_{I, B}(0,0)=1 \\
& J_{I, B}(1,1)=I(1,1)=1 \\
& J_{I, B}(1,0)=I(1,0)=0
\end{aligned}
$$

since $I$ is a fuzzy implication. Thus $J_{I, B}$ satisfies all axioms in Definition 2.1. 
Please note that if a function $J_{I, B}$ defined by (7) is a fuzzy implication, then $I$ satisfies also the identity principle (IP). Indeed, (2) holds in this case, so $J_{I, B}(x, 1)=1$ for all $x \in[0,1]$. But by Proposition 3.1 we know that $J_{I, B}(x, 1)=I(x, x)$ thus $I(x, x)=1$ for all $x \in[0,1]$.

What is interesting, the identity principle (IP) is not sufficient for being the $J_{I, B}$ defined by (7) a fuzzy implication, as the following example shows.

Example 3.5. Let us consider the nilpotent minimum t-norm

$$
T_{\mathbf{n M}}(x, y)= \begin{cases}0, & \text { if } x+y \leq 1 \\ \min \{x, y\}, & \text { elsewhere }\end{cases}
$$

and the residual implication to $T_{\mathbf{n M}}$ i.e., the Fodor implication given by the formula

$$
I_{\mathbf{F D}}(x, y)= \begin{cases}1, & \text { if } x \leq y \\ \max \{1-x, y\}, & \text { elsewhere }\end{cases}
$$

The $I_{\mathbf{F D}}$ satisfies (IP) as the R-implication generated from a left-continuous t-norm (see [3, Theorem 2.5.7]). But $J_{I_{\mathbf{F D}}, T_{\mathbf{n M}}}$ given by the formula

$$
\begin{aligned}
& J_{I_{\mathbf{F D}}, T_{\mathbf{n M}}}(x, y)= \\
& \quad= \begin{cases}1 & \text { if } x=0 \text { or } x \leq y \text { and } x+y>1, \\
1-x & \text { if } x>0 \text { and } x+y \leq 1, \\
y & \text { elsewhere, }\end{cases}
\end{aligned}
$$

is not a fuzzy implication since it is not a nonincreasing function with respect to the first variable. For the plot of this function see Figure 1.

Now, let us consider a few more examples.

Example 3.6. Let $B=T_{D}$ denote the smallest semicopula (3). Then

$$
J_{I, T_{D}}(x, y)= \begin{cases}I(1, y) & \text { if } \quad x=1 \\ I(x, x) & \text { if } y=1 \\ I(x, 0) & \text { elsewhere }\end{cases}
$$

is a fuzzy implication if and only if $I$ satisfies (IP) and $I\left(1^{-}, 0\right) \geq I\left(1,1^{-}\right)$, where the value $I\left(x^{-}, y^{-}\right)$ denotes the left-hand limit. It happens rather rarely, e.g. if $I=I_{T_{D}}$ is a residual implication generated by drastic t-norm $T_{D}$, where

$$
I_{T_{D}}(x, y)=\left\{\begin{array}{lll}
1 & \text { if } & x<1 \\
y & \text { if } & x=1
\end{array}\right.
$$

Example 3.7. Now let us consider the strongest semicopula $B=T_{M}$ given by (3). Then

$$
J_{I, T_{M}}(x, y)= \begin{cases}I(x, x) & \text { if } x \leq y \\ I(x, y) & \text { elsewhere }\end{cases}
$$

is a fuzzy implication for any fuzzy implication $I$ which satisfies the identity principle (IP). Indeed, if
$I\left(x_{0}, x_{0}\right)<1$ for some $x_{0} \in(0,1)$, then in particular $J_{I, T_{M}}\left(x_{0}, 1\right)<1$, which contradicts (2). Moreover, if $I$ satisfies (IP), then from the monotonicity $1=$ $I(x, x) \leq I(x, y) \leq 1$, so $I(x, y)=1$ for all $x \leq y$. Thus

$$
J_{I, T_{M}}=I
$$

when $I$ satisfies (IP).

Example 3.8. Let us consider the Gödel implication $I=I_{\mathbf{G D}}$, i.e. the residual of the largest t-norm (semicopula) $T_{M}$ given by (3), where

$$
I_{\mathbf{G D}}(x, y)= \begin{cases}1 & \text { if } x \leq y \\ y & \text { elsewhere }\end{cases}
$$

Then

$$
J_{I_{\mathbf{G D}}, B}(x, y)=\left\{\begin{array}{lll}
1 & \text { if } & x=B(x, y), \\
B(x, y) & \text { if } & x>B(x, y) .
\end{array}\right.
$$

is a fuzzy implication if and only if $B(x, y)=$ $\min \{x, y\}$, so $J_{I_{\mathbf{G D}}, B}(x, y)=I_{\mathbf{G D}}$. Indeed, let us assume that $J_{I_{\mathbf{G D}}, B}$ is a fuzzy implication for some semicopula $B$. We know that if $x>y$, then $B(x, y) \leq B(1, y)=y<x$ thus for any fixed $y_{0} \in[0,1)$ we have

$$
J_{I_{\mathbf{G D}}, B}\left(x, y_{0}\right)=B\left(x, y_{0}\right)
$$

for all $x>y_{0}$. As $B$ is semicopula, on this segment $B\left(\cdot, y_{0}\right)$ it is non-decreasing. But as we have a fuzzy implication, it satisfies also (I1), so it is nonincreasing with respect to the first variable. Hence $B\left(\cdot, y_{0}\right)$ is a constant function for all $x>y_{0}$. But $B\left(1, y_{0}\right)=y_{0}$ and thus

$$
B\left(x, y_{0}\right)=\min \left\{x, y_{0}\right\}
$$

for any fixed $y_{0}$ and all $x \in[0,1]$. Therefore

$$
\begin{aligned}
J_{I_{\mathbf{G D}}, T_{M}}(x, y) & =\left\{\begin{array}{lll}
1 & \text { if } & x=\min \{x, y\} \\
\min \{x, y\} & \text { if } & x>\min \{x, y\}
\end{array}\right. \\
& = \begin{cases}1 & \text { if } x \leq y \\
y & \text { elsewhere }\end{cases} \\
& =I_{\mathbf{G D}}(x, y)
\end{aligned}
$$

in this case again.

Proposition 3.9. Let $B=T$ denote a strict $t$ norm and let $I=I_{T}$ be a residual implication generated by $T$. Then

$$
J_{I_{T}, T}(x, y)= \begin{cases}1 & \text { if } x=0 \text { or } y=1 \\ y & \text { elsewhere. }\end{cases}
$$

Proof. We know that each continuous Archimedean t-norm $T$ could be expressed as

$$
T(x, y)=f^{-1}(\min \{f(x)+f(y), f(0)\}),
$$




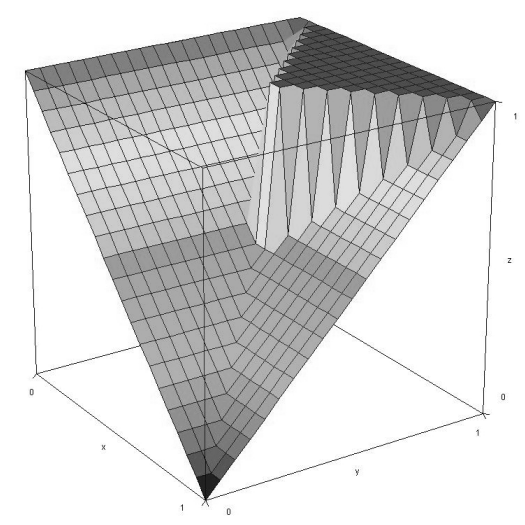

Figure 1: The $J_{I_{\mathbf{F D}}, T_{\mathbf{n M}}}$ function (see Example 3.5)

for all $x, y \in[0,1]$, where $f:[0,1] \rightarrow[0, \infty]$, called an additive generator, is a continuous, strictly decreasing function with $f(1)=0$, which is uniquely determined up to a positive multiplicative constant. It is also known (see [10]) that

$I_{T}(x, y)=f^{-1}(\max \{f(y)-f(x), 0\}), \quad x, y \in[0,1]$,

with the same $f$ (see [3, Theorem 2.5.21]).

Moreover, $T$ is strict if and only if its generator $f$ satisfies $f(0)=\infty($ see $[3$, Remark 2.1.7]). Hence, for a strict $T$ norm we have

$$
T(x, y)=f^{-1}(f(x)+f(y)), \quad x, y \in[0,1],
$$

and we obtain

$$
\begin{aligned}
J_{I_{T}, T} & (x, y)=I_{T}(x, T(x, y)) \\
& =I_{T}\left(x, f^{-1}(f(x)+f(y))\right) \\
& =f^{-1}(\max \{(f(x)+f(y))-f(x), 0\}) \\
& =f^{-1}(\max \{f(y), 0\}) .
\end{aligned}
$$

If $f(y)>0$ then the above expression is equal to $f^{-1}(f(y))=y$, and if $f(y)=0$ then $f^{-1}(0)=1$ and it happens if $y=1$. Of course, we also have $J_{I_{T}, T}(0, y)=1$, so finally we obtain

$$
J_{I_{T}, T}(x, y)= \begin{cases}1 & \text { if } \quad x=0 \text { or } y=1 \\ y & \text { elsewhere }\end{cases}
$$

which is the desired assertion.

Let us recall that a fuzzy implication is said to satisfy the left neutrality property if

$$
I(1, y)=y, \quad y \in[0,1]
$$

Please, note that the implication (9) discussed in the above proposition is the smallest fuzzy implication satisfying the left neutrality principle (NP).

Example 3.10. If the t-norm $T$ is nilpotent we have a slightly more general situation than that con- sidered in the last proof, i.e.

$$
\begin{aligned}
& J_{I_{T}, T}(x, y)=I_{T}(x, T(x, y)) \\
& \quad=I_{T}\left(x, f^{-1}(\min \{f(x)+f(y), f(0)\})\right) \\
& \quad=f^{-1}(\max \{\min \{f(x)+f(y), f(0)\}-f(x), 0\}) \\
& \quad=f^{-1}(\max \{\min \{f(y), f(0)-f(x)\}, 0\}) \\
& \quad=f^{-1}(\min \{f(y), f(0)-f(x)\}) \\
& \quad=\max \left\{y, f^{-1}(f(0)-f(x))\right\} .
\end{aligned}
$$

In particular case of the Eukasiewicz t-norm

$$
T_{\mathbf{L K}}=\max \{x+y-1,0\},
$$

with the additive generator $f(x)=1-x$, we obtain

$$
J_{I_{T_{\mathbf{L K}}}, T_{\mathbf{L K}}}(x, y)=\max \{1-x, y\}=I_{\mathbf{K D}}(x, y),
$$

known as the Kleene-Dienes implication (see [3]).

Keeping in mind the above mentioned examples it seems to be interesting to characterize both fuzzy implications $I$ and semicopulas $B$ so that the resulting functions $J_{I, B}$ would be also fuzzy implications. Therefore, let us define the following two sets.

For a fixed semicopula $B$ let

$$
\mathcal{J}_{B}=\left\{I \in \mathcal{F} \mathcal{I} \mid J_{I, B} \in \mathcal{F} \mathcal{I}\right\}
$$

denote a subfamily of fuzzy implications for which $J_{I, B}$ would be a fuzzy implication.

Similarly, for any fixed fuzzy implication $I$ let

$$
\mathcal{B}_{I}=\left\{B \in \mathcal{S C} \mid J_{I, B} \in \mathcal{F} \mathcal{I}\right\}
$$

denote a subfamily of semicopulas for which $J_{I, B}$ would be a fuzzy implication.

Example 3.11. Let $I_{S, N}(x, y)=S(N(x), y)$ be an $(\mathrm{S}, \mathrm{N})$-implication (see $[3])$. Moreover, let $\mathcal{T}$ denote a family of all t-norms. Then

$$
\mathcal{B}_{I_{S, N}}=\left\{T \in \mathcal{T} \mid J_{I_{S, N}, T} \in \mathcal{F} \mathcal{I}\right\}
$$

i.e., $\mathcal{B}_{I_{S, N}}$ with restriction to t-norms, yields implications of the following form

$$
J_{I_{S, N}, T}(x, y)=S(N(x), T(x, y)),
$$


which are the so-called QL-operation generated by the triple $(T, S, N)$. It is well-known that not all QL-operations are fuzzy implications (see [3, Lemma 2.6.5]). But, e.g., if $T$ is the minimum t-norm $T_{M}$, then the QL-operation generated by $\left(T_{M}, S, N\right)$ is a fuzzy implication.

By Proposition 3.2 we know that $J_{I_{\mathrm{GG}}, C}$ is a probabilistic implication for any copula $C$. Now let us consider a finite set of copulas $C_{1}, \ldots, C_{n}$. Let us adopt the following notation

$$
\begin{aligned}
& \vee C_{i}(x, y)=\max \left\{C_{1}(x, y), \ldots, C_{n}(x, y)\right\} \\
& \wedge C_{i}(x, y)=\min \left\{C_{1}(x, y), \ldots, C_{n}(x, y)\right\} .
\end{aligned}
$$

It is worth noting that both $\vee C_{i}$ and $\wedge C_{i}$ may not be copulas but they are semi-copulas. However, the following theorems hold.

Proposition 3.12. Let $I=I_{\mathbf{G G}}$ be the Goguen implication. If $J_{I_{\mathrm{GG}}, C_{i}}$ is a fuzzy implication for each copula $C_{1}, \ldots, C_{n}$ then both $J_{I_{\mathrm{GG}}, \vee C_{i}}$ and $J_{I_{\mathrm{GG}}, \wedge C_{i}}$ are fuzzy implications.

Proposition 3.13. Let $I=I_{\mathbf{L K}}$ be the Lukasiewicz implication and let $C_{1}, \ldots, C_{n}$ denote a set of copulas. Then both $J_{I_{\mathbf{L K}}, \vee C_{i}}$ and $J_{I_{\mathbf{L K}}, \wedge C_{i}}$ are fuzzy implications.

In fact, we may even formulate a more general theorem by considering not only finite systems of copulas but any nonempty set of copulas. Actually, it can be shown (see [12]) that for any nonempty set of quasi-copulas $\mathcal{U} \subset \mathcal{Q C}$ functions $\underline{\mathcal{U}}, \overline{\mathcal{U}}:[0,1]^{2} \rightarrow$ $[0,1]$ defined as follows

$$
\begin{aligned}
& \underline{\mathcal{U}}(x, y)=\inf \{Q(x, y) \mid Q \in \mathcal{U}\}, \\
& \overline{\mathcal{U}}(x, y)=\sup \{Q(x, y) \mid Q \in \mathcal{U}\},
\end{aligned}
$$

are quasi-copulas, i.e. $\underline{\mathcal{U}}, \overline{\mathcal{U}} \in \mathcal{Q C}$. Therefore, assuming that $\mathcal{U}$ denotes a nonempty set of copulas and keeping in mind that any copula is a quasicopula, we obtain stronger versions of the above propositions.

Proposition 3.14. Let $I=I_{\mathbf{G G}}$ be the Goguen implication. If $J_{I_{\mathrm{GG}}, C}$ is a fuzzy implication for each copula $C \in \mathcal{U}$ then both $J_{I_{\mathrm{GG}}, \underline{\mathcal{U}}}$ and $J_{I_{\mathrm{GG}}, \overline{\mathcal{U}}}$ are fuzzy implications.

Proposition 3.15. Let $I=I_{\mathbf{L K}}$ be the Lukasiewicz implication and let $\mathcal{U}$ denote a set of copulas. Then both $J_{I_{\mathbf{L K}}, \underline{\mathcal{U}}}$ and $J_{I_{\mathbf{L K}}, \overline{\mathcal{U}}}$ are fuzzy implications.

\section{Conclusions}

We have introduced a new construction method for implications based on a fuzzy implication $I$ and a semicopula $B$. The resulting implication $J_{I, B}$ is an extension of the boolean implication, however, its monotonicity in the first coordinate may fail, and thus it need not be a fuzzy implication, in general.
Our method generalizes the probabilistic implications (they also need not be fuzzy implications, in general) and the probabilistic S-implications. Several distinguished fuzzy implications can be represented in the form $J_{I, B}$, for example the KleeneDienes implication, of QL-implications. Our proposal opens several problems, especially a full characterization of the classes $J_{B}$ (when a semicopula $B$ is fixed) and $B_{I}$ (when a fuzzy implication $I$ is fixed).

\section{References}

[1] M. Baczyński, G. Beliakov, H. Bustince and A. Pradera, editors, Advances in Fuzzy Implication Functions, Studies in Fuzziness and Soft Computing, Vol. 300, Springer, Berlin Heidelberg, 2013.

[2] M. Baczyński, P. Grzegorzewski and W. Niemyska, Laws of contraposition and law of importation for probabilistic implications and probabilistic S-implications, In A. Laurent, O. Strauss, B. Bouchon-Meunier and R.R. Yager, editors, Information Processing and Management of Uncertainty in KnowledgeBased Systems, 15th International Conference, IPMU 2014, Montpellier, France, July 15-19, 2014, Proceedings, Part I, Communications in Computer and Information Science 442, pages 158-167, Springer International Publishing, 2014.

[3] M. Baczyński and B. Jayaram, Fuzzy Implications, Studies in Fuzziness and Soft Computing, Vol. 231, Springer, Berlin Heidelberg, 2008.

[4] A. Dolati, J.F. Sánchez and M. Úbeda-Flores, A copula-based family of fuzzy implication operators, Fuzzy Sets and Systems 211:55-61, 2013.

[5] F. Durante and C. Sempi, Semicopulae, Kybernetika, 41:315-328, 2005.

[6] P. Grzegorzewski, Probabilistic implications, In S. Galichet, J. Montero and G. Mauris, editors, Proc. of the rth Conference on European Society for Fuzzy Logic and Technology (EUSFLAT-2011) and LFA-2011 Aix-lesBains, France, July 18-22, 2011, Advances in Intelligent Systems Research, Vol. 1, pages 254-258, Atlantis Press, 2011.

[7] P. Grzegorzewski, On the properties of probabilistic implications, In P. Melo-Pinto, P. Couto, C. Serôdio, J. Fodor and B. De Baets, editors, Eurofuse 2011, Workshop on Fuzzy Methods for Knowledge-Based Systems, Advances in Intelligent and Soft Computing, Vol. 107, pages 67-78, Springer-Verlaq, 2011.

[8] P. Grzegorzewski, Survival Implications, In S. Greco, B. Bouchon-Meunier, G. Coletti, M. Fedrizzi, B. Matarazzo and R.R. Yager, editors, Advances in Computational Intelligence, 
Part II (Proc. 14th International Conference, IPMU 2012, Catania, Italy, July 9-13, 2012),

Communications in Computer and Information Science 298, pages 335-344, Springer-Verlag, 2012.

[9] P. Grzegorzewski, Probabilistic implications, Fuzzy Sets and Systems, 226:53-66, 2013.

[10] E.P. Klement, R. Mesiar and E. Pap, Triangular norms, Kluwer, Dordrecht, 2000.

[11] M. Miyakoshi and M. Shimbo, Solutions of composite fuzzy relational equations with triangular norms, Fuzzy Sets and Systems, 16:5363, 1985.

[12] R.B. Nelsen, An Introduction to Copulas, 2nd Edition, Springer, New York, 2006. 